\title{
Dynamics and temperature of droplets impacting onto a heated wall
}

\author{
G. Castanet*, T. Liénart, F. Lemoine \\ LEMTA, Nancy-Université, CNRS, 2 Avenue de la Forêt de Haye, BP 160, 54504 Vandœuvre-lès-Nancy, France
}

\begin{abstract}
A B S T R A C T
This paper presents results of an experimental investigation of water droplet impacts onto a smooth heated plate made of nickel. A high-speed camera is used to observe the impact regimes (rebound, splashing, and deposition of a liquid film) for a large set of impact conditions (wall temperature, droplet incidence angle, velocity and size). The observations help specifying the conditions of existence of the regimes. In a second part of the paper, the emphasis is placed on the droplet heating during an interaction with the heated wall. The droplet temperature is measured with the help of the two-colour laser-induced fluorescence thermometry in the case of the different impact regimes. The droplet change in temperature during an impact is found to be dependent on the normal velocity but not on the wall temperature when it exceeds the Leidenfrost temperature.
\end{abstract}

\section{Drop impact}

Laser-induced fluorescence

Temperature measurement

Spray cooling

\section{Introduction}

Liquid cooling is unavoidable in application areas where the required power dissipation is very large. Possible liquid cooling technologies include immersion flow boiling; spray cooling, jet impingement cooling. While pool boiling and jet impingement techniques have satisfactorily provided high heat dissipation rates, they have generally failed to insure uniform and controlled cooling because of large spatial variations in surface heat flux.

In the steel industry, heat treatment of the alloys consists in a heating and cooling process designed to alter the metallurgical microstructure in order to obtain given mechanical properties. An ideal quench is one that proceeds at an infinitely fast rate since this would avoid any massive precipitation of the alloy constituents along grain boundaries, which results generally in an alloy with poor strength and poor hardness [1]. However, quench rate is slowed by the moderate heat transfer efficiency of the film boiling regime occurring at the beginning of the quench. At extremely high surface temperatures (above the Leidenfrost temperature), an insulating vapour film quickly develops at the contact between the liquid and the solid surface [1]. Unfortunately, much of the detrimental coalescence of solutes along the grain boundaries occurs at temperatures associated with the film boiling regime. Up to now, most of heat treatments are still performed by means of high velocity impinging jets.

However, researches on heat transfers in liquid jet impingement reveals very high heat transfer rates at the stagnation point of the jet, whereas the radial region is characterized by more moderate heat transfer coefficients [2]. The irregular cooling by liquid

\footnotetext{
* Corresponding author. Tel.: +3303 835956 46; fax: +330383595544.

E-mail address: guillaume.castanet@ensem.inpl-nancy.fr (G. Castanet).
}

jets is a serious drawback that would be limited if spray cooling could be used instead. On the other hand, imposed environmental and economic constraints have resulted in greater demands for material with better recyclability and lower cost. Significant quantities of liquid coolant could be saved with spray cooling. Heat transfer rates much higher than can be attained in pool boiling are possible with sprays since the mass transfers at the wall are enhanced. However, while spray quenching offers potential enhancement compared to other methods, its immediate integration is still limited because of incomplete understanding of the complex fluid flow and heat transfer characteristics of sprays interacting with heated surfaces.

The impingement of droplets on solid surfaces has received a considerable attention throughout the decades. Many studies have been focussed on the impact conditions that can occur at high or low speed with deep or shallow liquid film, on hot or cold solid surfaces, etc. Depending on these conditions, qualitatively different behaviours can occur: the drops can spread over the solid surface, they can splash by creating a crown and droplet rebounds are also possible particularly on hot surfaces. Extensive experimental investigations were carried out to determine the parameters influencing the behaviour of a single drop impact in order to characterize their respective influence. Some of these parameters describe the geometry and the dynamic of the drops (velocity and diameter $[3,4]$, angle of incidence [5]), some relate to the physical properties of the liquid (viscosity [6], surface tension [4,6], etc.) or the solid surface (roughness [4], wall effusivity [7], etc.). Experimental results are generally analyzed on the basis of correlations based on dimensionless numbers characterizing the relative magnitude of the forces acting on the impinging droplet and estimated with the physical properties of the liquid before impact, i.e. Reynolds, Weber and Ohnesorge numbers. However, these analyses still 


\begin{tabular}{|c|c|c|c|}
\hline \multicolumn{4}{|c|}{ Nomenclature } \\
\hline \multicolumn{2}{|c|}{ Latin } & \multicolumn{2}{|c|}{ Symbols } \\
\hline C & fluorescent tracer concentration & $\alpha$ & angle of incidence \\
\hline$D$ & droplet diameter & $\varepsilon$ & thermal effusivity \\
\hline$f$ & injection frequency & $\lambda$ & heat conductivity or wavelength \\
\hline I & light intensity & $\rho$ & density \\
\hline \multicolumn{2}{|c|}{$\begin{array}{c}K=W e \mathrm{Oh}^{-0.4} \text { dimensionless number introduced to describe the } \\
\text { outcome of an impact }\end{array}$} & $\sigma$ & surface tension \\
\hline$L$ & distance between the droplets & \multicolumn{2}{|c|}{ Subscripts } \\
\hline $\mathrm{Oh}$ & Ohnesorge number & $\mathrm{b}$ & boiling point \\
\hline$R a$ & averaged roughness & $\mathrm{n}$ & normal to the wall \\
\hline$T$ & temperature & 1 & liquid phase \\
\hline V & droplet velocity & $\mathrm{L}$ & Leidenfrost conditions \\
\hline We & Weber number & & \\
\hline
\end{tabular}

suffer from limitations, since they generally take into account a limited number of parameters. For instance, the temperature of the wall, or the level of droplet subcooling is seldom taken into account although many studies have described that dynamic regimes are modified in the case of high-temperature surfaces. It is in particular well-established that a transition occurs above the Leidenfrost temperature from ideally bouncing droplets to droplets that break-up [8-10]. Wachters and Westerling [10] studied the impact of water droplets impinging on a horizontal surface at $400{ }^{\circ} \mathrm{C}$. The outcomes of the impact falls into three general categories depending on the droplet Weber number. For $W e<30$, the droplet spreads into a flat disk but the liquid finally regrouped itself under the action of surface tension forces and rebounded off the heated surface. For intermediate Weber numbers $(30<W e<80)$, the droplet undergoes a similar behaviour except that the droplet split into a large droplet and a small satellite droplet. Finally for $W e>80$, the droplet breaks into several small droplets. Nonetheless, despite an increasing amount of experimental data, full knowledge of impact regimes is still lacking and only a few publications have been reported where the authors tried to build up a complete regime map for the impingement process on hot surfaces. Amiel [11] performed numerous experiments on ethanol droplets. Impacts regimes (rebound, splashing, film deposition, etc.) were shown to enter within a diagram of the wall temperature versus the Weber number. García Rosa et al. [12] gave explicit expressions for the position of the limits between the different impacts regimes based on these observations.

Heat transfers were generally characterized by thermocouples embedded in the sample thickness. It has been possible to monitor the history of the surface temperature at the location of the droplet impingement [13]. The heat flux to the wall was also estimated when the experimental set-up was designed to ensure a onedimensional heat flux along the instrumented section containing a set of thermocouples. All these measurements provided valuable data to quantify the efficiency of the cooling under different experimental conditions, like single droplet streams or multiple droplet stream arrays [1]. However, this kind of measurement has only a limited interest when it comes to understand the phenomena occurring within the liquid phase. To our knowledge, no data is available in the literature to model the temperature increase of the droplets when they interact with a hot surface. The respective importance of the sensible heat and the heat of vapourization is therefore still unclear. Additionally, in the modelling of the droplet impacts, physical properties of the liquid are generally calculated on the basis of the injection temperature of the liquid. However, the liquid temperature is likely to vary from the injection to the impact due to the heating of the droplets which can occur either at the contact of the solid surface or in the hot air plume created by the heated surface. A measurement of the droplet temperature would also help to improve the modelling of the droplet impact by accounting for the thermal variation of the physical properties of the liquid throughout the impingement process. This paper relates one of the first attempts to measure the temperature of the droplet impinging onto a hot solid surface. The case of water droplets impacting onto a smooth heated plate made of nickel is considered. After a description of the different impact regimes depending on the experimental conditions, measurements of the droplets will be presented. These measurements are applied to the case of a boiling liquid film, the rebound and splashing regimes. In these two latter, the wall temperature is higher than the Leidenfrost temperature.

\section{Description of the experimental set-up}

In order to study droplet/wall interaction phenomena, an experimental set-up was specifically designed. This section describes the equipment used for the droplet generation as well as the heated plate.

\subsection{The heated plate}

The heated plate is a parallelepiped $(2 \times 2 \times 10 \mathrm{~cm})$ which is made of an alloy containing over $99 \%$ of nickel. A cylindrical hole is drilled in the centre of the plate in order to insert an electrical resistance of $110 \Omega$ (Fig. 1). The resistance diameter ( $7 \mathrm{~mm}$ ) and its length match exactly the dimensions of the hole, so that an optimal contact is obtained between the nickel plate and the resistance. The plate is then heated with the help of the energy dissipated into the resistance by Joule effect and to minimize heat losses, the plate lays on a ceramic insulator. A thermocouple is inserted in the core of the heated plate to characterize its temperature. The thermocouple is also connected to a power regulator that enables to adjust the voltage supplied to the resistance in order to maintain a setpoint temperature. Since nickel is an efficient heat conductor $\left(\lambda=50 \mathrm{~W} \mathrm{~m}^{-1} \mathrm{~K}^{-1}\right)$, a rather homogeneous distribution of the temperature inside of the plate is expected. The wall temperature has been measured using an infrared camera in the absence of droplets. In the middle of the plate, where the impacts take place, the wall temperature is rather uniform. Moderate variations of the temperature in the range of $10^{\circ} \mathrm{C}$ can be observed when the wall temperature is $330^{\circ} \mathrm{C}$ (Fig. 2).

To study the droplet impingement, the roughness of the wall is also a very important parameter. The upper surface of the plate on which the droplet are impacting, has been polished so as to obtain 


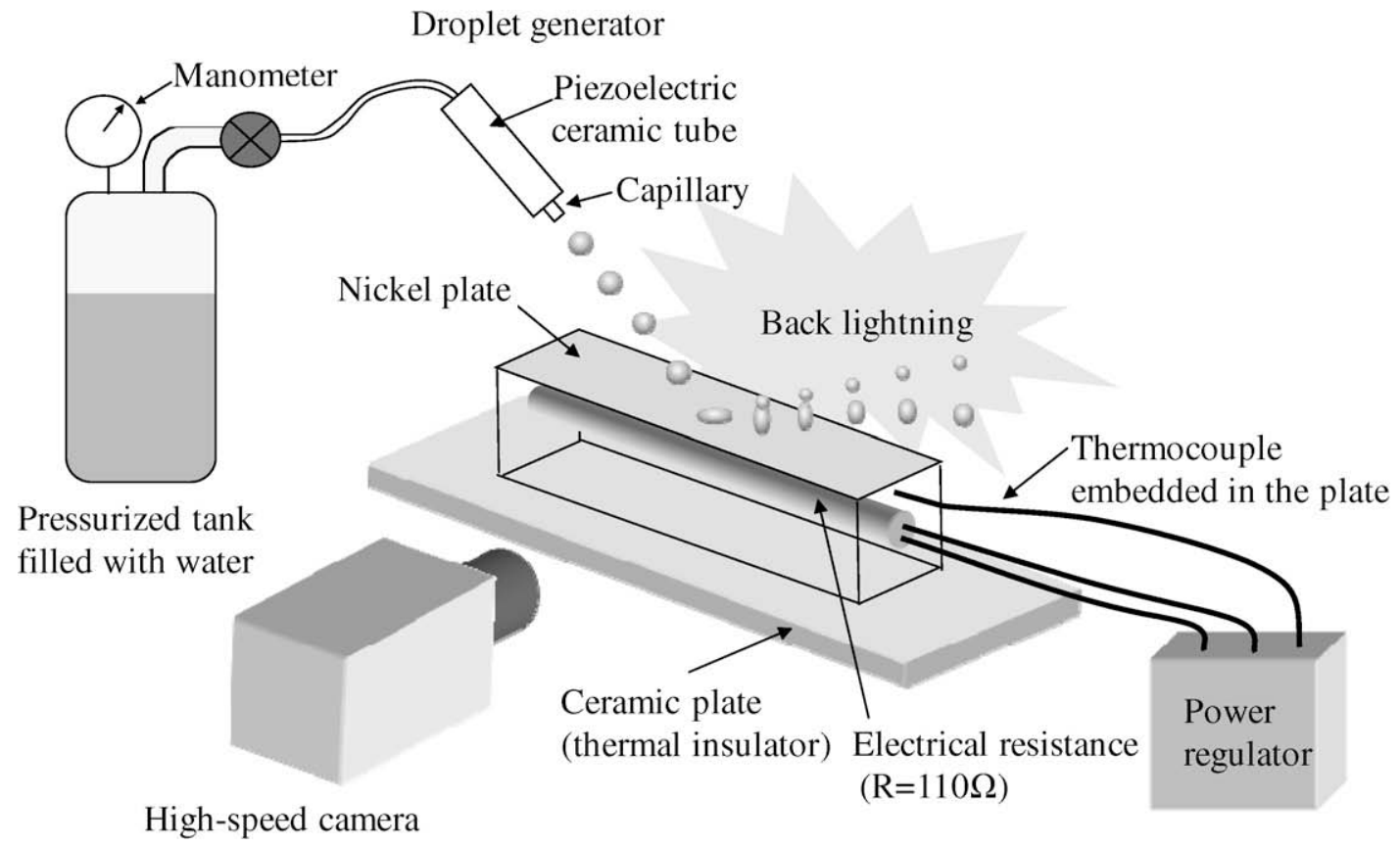

High-speed camera

Fig. 1. Schematic view of the experimental set-up and the optical layout for shadow imagery.

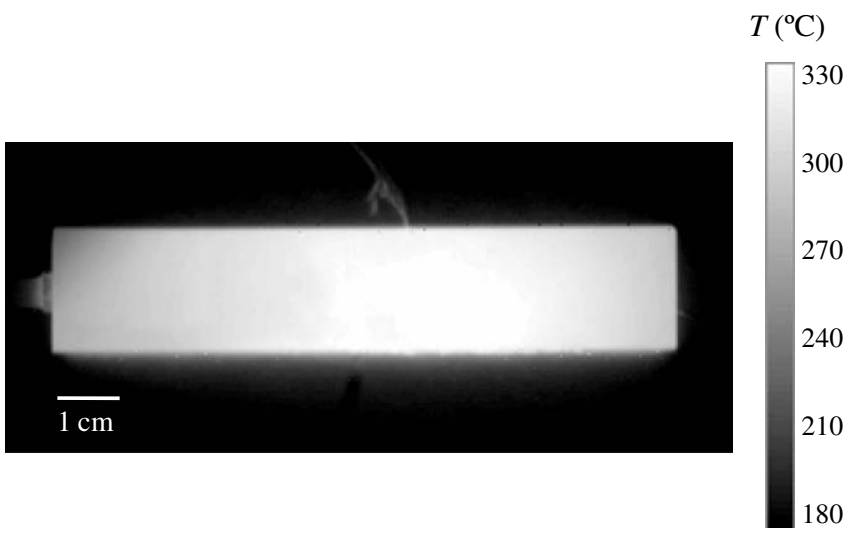

Fig. 2. Distribution of the wall temperature obtained by means of an infrared camera $\left(T_{\mathrm{w}}\right.$ is about $\left.330^{\circ} \mathrm{C}\right)$.

a smooth surface. Roughness profiles were recorded in different places of the wall and the average roughness $R a$ was estimated at about $0.5 \mu \mathrm{m}$. The oxidation of pure nickel remains limited when the wall temperature does not exceed $500{ }^{\circ} \mathrm{C}$ like in the present study. A very light brown colour can appear on some area of the nickel surface (related to impurities) whereas nickel oxide is somewhat green and gray. When the droplets impinge onto the wall repeatedly at the same location, a kind of metal fatigue can be noticed at the impact location probably due to thermal constraints. After a few minutes, droplet behaviours may become unpredictable (for example an unsteady bouncing angle or the presence of splashing under conditions corresponding normally to a rebound) which seems to indicate that the surface roughness is certainly changed. To avoid this problem, in practice, the plate is moved a few millimeters before any new measurement.

\subsection{The droplet generator}

The monodisperse generator was manufactured in University of Bremen, Germany [14]. The most important parts of the droplet generator are a piezoelectric ceramic tube and a glass capillary which is encapsulated into the ceramic tube. The piezoelectric ceramic is driven by an electric pulse generator. Depending on the voltage applied to the piezoelectric ceramic, the inner diameter of the piezoelectric tube widens or narrows and the contractions are transmitted to the glass capillary. In the so-called droplet-ondemand mode, the pulse duration and amplitude are adjusted so that the contraction of the capillary is large enough for a single droplet to be ejected from the nozzle. To produce a continuous chain of monosized droplets, the droplet generator is operated with an excess pressure (typically 1 bar). If a frequency is applied to the driving voltage of the piezoelectric ceramic, a regular excitation of the liquid jet is achieved. For specific frequencies, the cylindrical liquid jet breaks-up into equally sized and periodically arranged droplets due to Rayleigh-type instabilities [15]. In the present study, the droplet generator is used to produce streams of monodisperse water droplets.

The droplet generator is placed about $5 \mathrm{~cm}$ from the impact location in order to work with very stable and coherent droplet streams. The generator can be rotated to obtain several angles of incidence.

\section{Measurement techniques}

\subsection{Droplet visualization system}

A high-speed (HS) camera is used to visualize the impingement process of individual droplet. The HS camera (Photron ultima APX$\mathrm{RS}$ ) is equipped with a 10 bits CMOS sensor that can provide up to $3000 \mathrm{fps}$ at full resolution $(1024 \times 1024$ pixels $)$ and $250,000 \mathrm{fps}$ at reduced resolution. The droplets are illuminated from behind using a white light source which enables to get very sharply contrasted images of the droplet contour by shadow imaging (Fig. 1). A macro-lens is mounted at the front of the camera to observe the droplet deformation and the possible occurrence of secondary droplets. The magnification factor is determined before the measurements by imaging a reference target, the size of which is known accurately. The images are then processed in order to determine the incident angle $\alpha$ of the impacts and the spacing $L$ between 
the impinging droplets. The latter is eventually used to estimate the velocity of the impinging droplets. Since the droplets are periodically arranged, their velocity is simplify given by $V=L \cdot f$, with $f$ denoting the injection frequency.

\subsection{Two-colour laser-induced fluorescence thermometry}

The main outlines of the two-colour laser-induced fluorescence thermometry are presented in this section. Additional features related to the techniques can be found in previous papers $[16,17]$. Water is previously seeded with a low concentration (a few $\mathrm{mg}$ / L) of rhodamine B. The fluorescence of rhodamine B is induced by the green line of an argon ion laser $(\lambda=514.5 \mathrm{~nm})$. The fluorescence spectrum is broadband and exhibits a temperature sensitivity which is strongly dependent on the wavelength [16]. The fluorescence intensity is given by [16]

$I_{f}(\lambda)=K_{\text {opt }}(\lambda) K_{\text {spec }}(\lambda) V_{\text {mes }} I_{0} C \mathrm{e}^{\beta(\lambda) / T}$

where $K_{\text {opt }}(\lambda)$ is an optical constant, $K_{\text {spec }}(\lambda)$ is a constant depending solely on the spectroscopic properties of the fluorescent tracer in its solvent (i.e. water), $I_{0}$ the laser intensity, $C$ the molecular tracer concentration, $T$ the absolute temperature, $V_{\text {mes }}$ is the measurement volume. The product $C \cdot V_{\text {mes }}$ is proportional to the number of fluorescence photons emitted by the rhodamine $\mathrm{B}$ molecules excited by the laser radiation and reaching the photodetector surface. $\beta(\lambda)$ features the temperature dependence of fluorescence intensity at the wavelength $\lambda$. In order to collect a large number of photons, the fluorescence signal is detected over a spectral band $\left[\lambda_{i 1} ; \lambda_{i 2}\right], i$ denoting the spectral band. The resulting signal can be expressed using second-order polynomials [17]

$\int_{\lambda_{i 1}}^{\lambda_{i 2}}=K_{\text {opt }}(\lambda) K_{\text {spec }}(\lambda) V_{\text {mes }} I_{0} C \mathrm{e}^{\beta(\lambda) / T} \mathrm{~d} \lambda=K_{\text {opti }} K_{\text {speci }} V_{\text {mes }} I_{0} C \mathrm{e}^{\frac{a_{i}}{T^{2}}+\frac{b_{i}}{T}}$

where $K_{\mathrm{opt}, i}$ and $K_{\mathrm{spec}, i}$ are the optical and spectroscopic constants related to the spectral band $i$. The parameters $a_{i}$ and $b_{i}$ are the temperature sensitivity coefficients for the spectral band $i$. Measuring the temperature of a moving, evaporating, and potentially deformed droplet requires to eliminate the influence of the parameters $C \cdot V_{\text {mes }}$ and $I_{0}$. The measurement volume is constantly changing when the droplet crosses the laser beam. This volume corresponds indeed to the triple intersection between the volume of excitation by the laser beam, the volume of detection by the receiving optics and the droplet. The intensity distribution of the laser inside the droplet depends on the droplet shape and on its position relatively to the beam due to the lensing of the droplet interface. In the case of an evaporating droplet, the concentration of the fluorescent tracer is likely to vary. In order to overcome these problems, the fluorescence intensity is detected over two spectral bands for which the temperature sensitivity is highly different. Selection of these spectral bands is optimized with a preliminary study of the influence of temperature on the fluorescence spectrum of rhodamine B dissolved in water. A first band [525-535 nm] and a second one centred at $580 \mathrm{~nm}$ with a bandwidth of $10 \mathrm{~nm}$ is a good trade-off between a sufficient signal intensity and a good temperature sensitivity. The ratio between both fluorescence intensities is given by

$R_{f}=\frac{I_{f 1}}{I_{f 2}}=\frac{K_{\text {opt } 1}}{K_{\text {opt2 }}} \frac{K_{\text {spec } 1}}{K_{\text {spec } 2}} \mathrm{e}^{\frac{a_{1}-a_{2}}{T^{2}}+\frac{b_{1}-b_{2}}{T}}$

This ratio is totally independent on the dimensions of the intersection between the droplet and the excitation and detection volumes. The influence of the local laser intensity and tracer concentration is also totally eliminated. Even if the fluorescence is almost proportional to the droplet volume and evolves therefore as a function of the droplet size. The ratio of the fluorescence intensity of the two spectral bands removes this dependence as well. This ratio depends exclusively on the temperature for a given tracer in a given solvent which is one the main limitation of the technique. The signal that is detected on each of the spectral bands should be subtracted from the background noise (obtained by switching off the laser). It is then possible to verify that the ambient light scattered by droplets is negligible (even non-existent).

The set of coefficients $\left(a_{1}-a_{2}\right)$ and $\left(b_{1}-b_{2}\right)$ is preliminarily determined in a temperature-controlled cell.

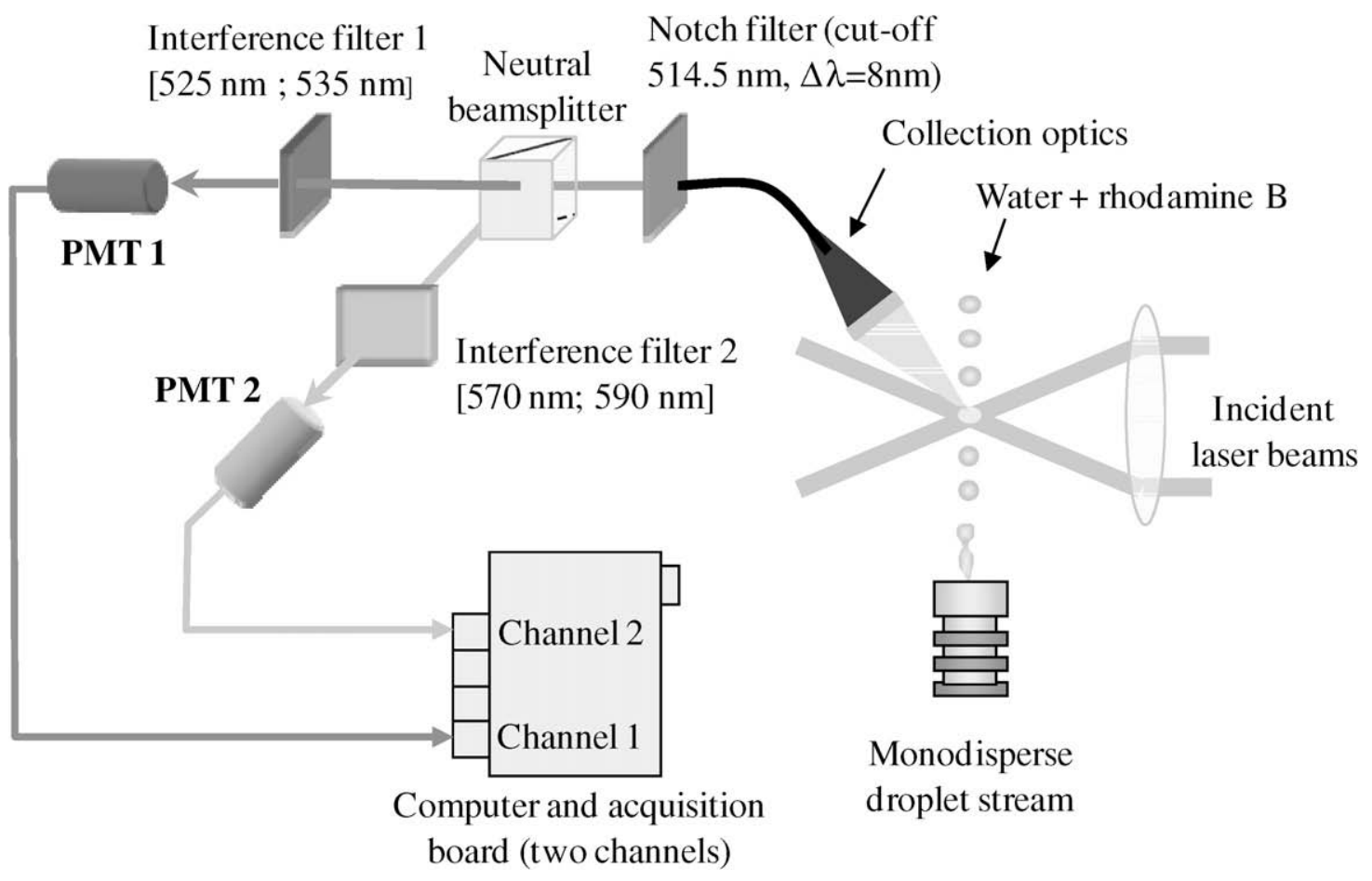

Fig. 3. Two-colour laser-induced fluorescence optical set-up. 
The use of a single reference point where temperature is known allows eliminating the optical and spectroscopic constants $K_{\mathrm{opt}}$ and $K_{\text {spec }}$, generally this reference is taken very near the injector exit. The excitation volume is formed by means of the beam system of a laser Doppler Anemometer (Fig. 3). It corresponds to an ellipsoid of revolution which is $1500 \mu \mathrm{m}$ long in the direction of the beams and $150 \mu \mathrm{m}$ long transversally (Fig. 9). A volume-averaged temperature can be obtained using an excitation volume with a diameter comparable to the droplet size and by averaging the fluorescence signal over the overall transit time of the droplet in the measurement volume [16]. The fluorescence signal is transmitted by an optical fibre to a set of beamsplitters and optical filters, which divide the fluorescence signal into the two spectral bands of interest. Mie scattering of the laser excitation wavelengths is eliminated using a high-pass filter with a high optical density at the laser wavelength.

The fluorescence signal is detected over the two spectral bands by means of two photomultiplier tubes equipped with two rapid pre-amplifiers. Since fluorescent molecules are distributed uniformly over the droplet volume, the photons collected by the detectors come from the interior of the droplet. In the present study, the beam diameter $(150 \mu \mathrm{m})$ is larger the droplet size (about $100 \mu \mathrm{m}$ ), therefore the measured temperatures can be safely considered as volume-averaged temperatures. Measurements are averaged over about 10,000 droplets crossing the measurement volume. Because of the injection frequency of $10 \mathrm{kHz}$, the duration of the measurement was in the order of one second (a little more in the case of a splashing). This number appears sufficient to reach the statistical convergence with a RMS less than $1{ }^{\circ} \mathrm{C}$ provided that the droplet stream was periodic. This accuracy is in principle not affected by the possible non-sphericity of the droplets or the presence of internal thermal gradients unlike the rainbow refractometry [18].

\section{Mapping of the impact regimes}

In this section, the emphasis is laid on the observation of the impact regimes. A large number of experiments were carried out in order to test the influence of the impact conditions such as the wall temperature, the droplet size and velocity. These experiments consisted in observing the outcome of an impact, i.e. whether it is a bouncing, a splashing or a deposition. While the frequency of the droplet injection was maintained at $10 \mathrm{kHz}$ throughout the experiments, the velocity of the droplets was increased from 4 to $13 \mathrm{~m} / \mathrm{s}$ and the droplet size from 90 to $200 \mu \mathrm{m}$. In addition, the angle of incidence of the droplet trajectory was also changed to investigate situations from low-angle to normal incidences. The wall was heated to reach temperatures ranging from 120 to $460{ }^{\circ} \mathrm{C}$. These temperatures are distributed around the static Leidenfrost temperature of water which is about $220^{\circ} \mathrm{C}$ for a sessile droplet set on a smooth surface [19]. Images corresponding to different impact regimes are presented in Fig. 4. The plate is smooth so that it behaves like a mirror that reflects the image of the droplets. For this reason, the symmetry of the droplets can be seen sometimes on the lower part of the pictures (Figs. 4-6). When the wall temperature is lower than the static Leidenfrost temperature or very close to it, the droplets spread completely over the wall surface (Fig. 4a and b). If the wall temperature is largely higher than the boiling temperature, the liquid evaporates very quickly and is heated to its boiling point (nucleate boiling regime). Bubbles of vapour are generated in the liquid film and secondary droplets are expelled from the bubbles explosion (Fig. 4b). When the wall temperature is closer to the boiling temperature of water (for example $T_{\mathrm{w}}=120^{\circ} \mathrm{C}$ ), no bubbles can be observed anymore (Fig. 4a). The continuous inflow
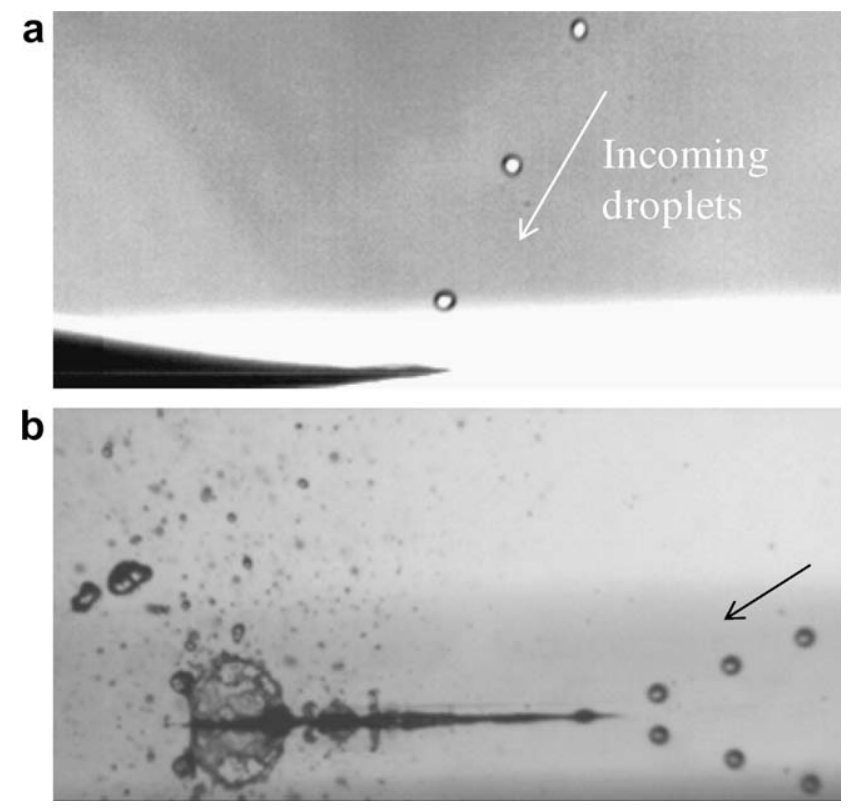

Fig. 4. Illustrations of the film deposition regime with and without the boiling of the liquid film ((a) $T_{\mathrm{w}}=120^{\circ} \mathrm{C}, D=111 \mu \mathrm{m}, V=6.9 \mathrm{~m} / \mathrm{s}, \alpha=64^{\circ}, f=10 \mathrm{kHz}$; (b) $\left.T_{\mathrm{w}}=120^{\circ} \mathrm{C}, D=150 \mu \mathrm{m}, V=5.2 \mathrm{~m} / \mathrm{s}, \alpha=21^{\circ}, f=10 \mathrm{kHz}\right)$.

of cold liquid droplets is then sufficient to prevent the liquid film from boiling. Furthermore, it has to be added that heat transfer
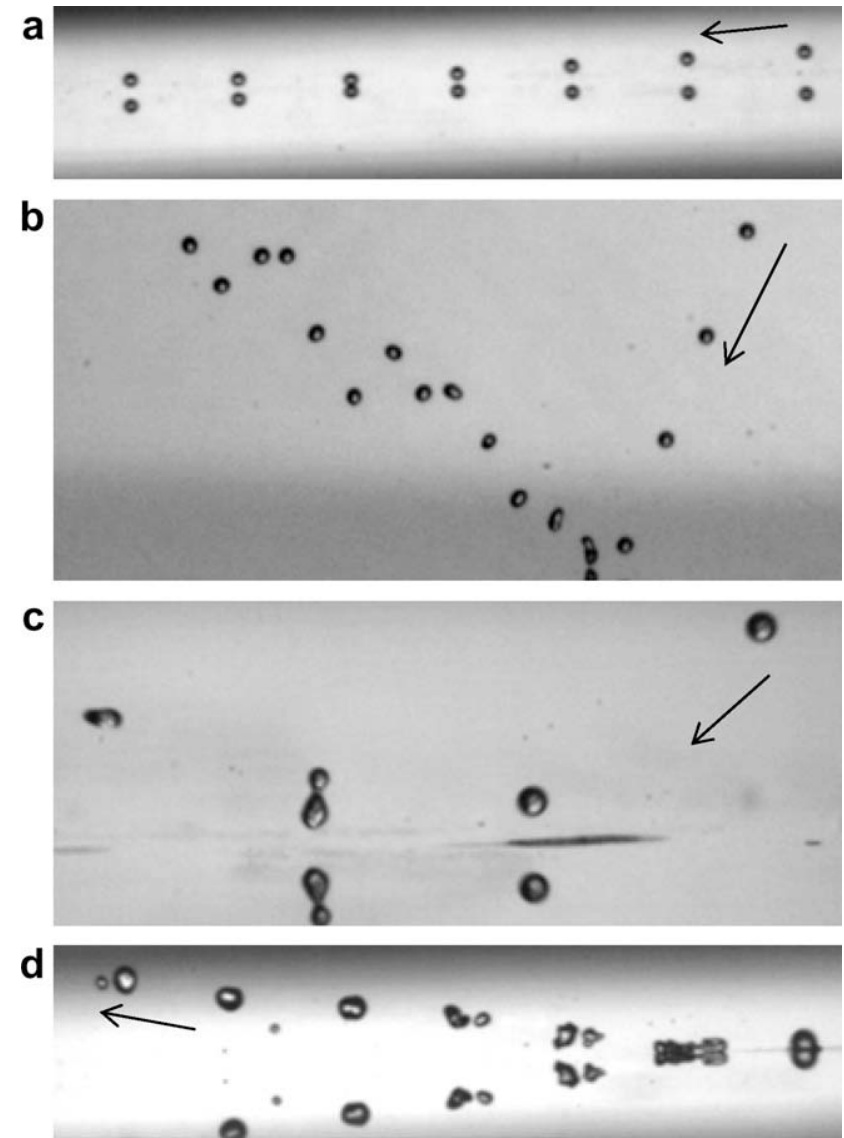

Fig. 5. Illustration of different cases of rebounds ((a) $T_{\mathrm{w}}=460{ }^{\circ} \mathrm{C}, D=102 \mu \mathrm{m}$, $V=7 \mathrm{~m} / \mathrm{s}, \quad \alpha=4^{\circ}, f=10 \mathrm{kHz} ; \quad$ (b) $T_{\mathrm{w}}=330^{\circ} \mathrm{C}, \quad D=104 \mu \mathrm{m}, \quad V=6 \mathrm{~m} / \mathrm{s}, \alpha=68^{\circ}$, $f=10 \mathrm{kHz} ; \quad$ (c) $T_{\mathrm{w}}=250^{\circ} \mathrm{C}, \quad D=125 \mu \mathrm{m}, \quad V=6.5 \mathrm{~m} / \mathrm{s}, \quad \alpha=36^{\circ}, f=10 \mathrm{kHz} ; \quad$ (d) $T_{\mathrm{w}}=405^{\circ} \mathrm{C}, D=145 \mu \mathrm{m}, V=10 \mathrm{~m} / \mathrm{s}, \alpha=29^{\circ}, f=10 \mathrm{kHz}$ ). 


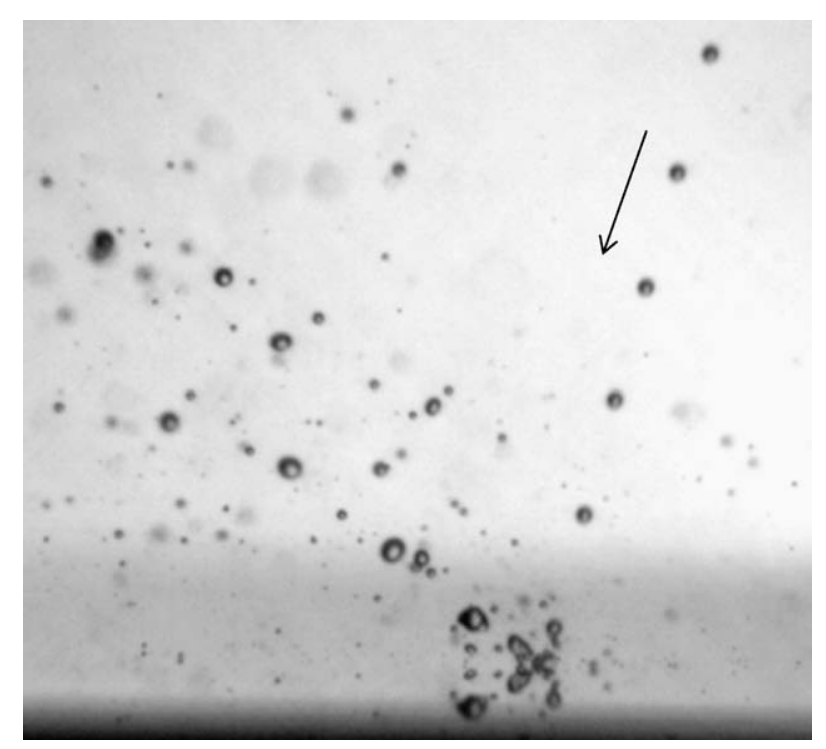

Fig. 6. Image illustrating the splashing regime $\left(T_{\mathrm{w}}=250{ }^{\circ} \mathrm{C}, D=115 \mu \mathrm{m}, V=7.2 \mathrm{~m} /\right.$ s, $\alpha=74^{\circ}, f=10 \mathrm{kHz}$ ).

tends to reduce locally the temperature of the wall surface which can be smaller than the one measured by the thermocouple in the wall thickness. When the bubble boiling deposition regime starts to disappear, the temperature of the wall surface is possibly already slightly below the boiling point of water.

When the wall temperature is higher than the Leidenfrost temperature, two main impact regimes can be observed: the bouncing and splashing regimes (Figs. 5 and 6). If the droplet velocity normal to the wall surface is sufficiently low, rebounds prevail. Rebounds can take on several appearances depending on the conditions. It can be a perfect rebound when the droplets are almost not deformed during the process (Fig. 5a). A very low velocity and a very high-temperature can be considered as stabilizing factors to obtain this kind of rebound since the vapour cushion is increased. In other cases of rebound, the droplets can lose their linear arrangement and be stretched to the break-up (Fig. 5b). Eventually, break-up can take place leading to a satellite droplet that may coalesce (Fig. 5c) or not (Fig. 5d) to form again a unique droplet. If the normal velocity keeps on being increased, then splashing occurs with emission of secondary droplets (Fig. 6).

The images were analyzed on the basis of dimensionless numbers. The Mundo number $K$, which is a combination between the Weber number and the Ohnesorge number can help to describe the droplet/wall interaction, since these two numbers are particularly relevant in the present situation. This number is defined by the following expression:

$$
\begin{aligned}
& K=W e O h^{-0.4} \\
& W e=\frac{\rho_{1} D V_{n}^{2}}{\sigma} \\
& O h=\frac{\mu_{1}}{\sqrt{\rho_{1} D \sigma}}
\end{aligned}
$$

The number $K$ was introduced first by Mundo [20] and was used successfully by Cossali [21] to describe the transition between the deposition and the splashing regime. It should be also emphasized that the Weber number is based on the normal component of the droplet velocity as stated in Eq. (5). The physical properties to evaluate the Weber and Ohnesorge numbers are estimated at the temperature of injection of the drops which was maintained around $20^{\circ} \mathrm{C}$. Additionally, a dimensionless tem- perature is introduced to describe the influence of the wall temperature. Its expression is based on the previous work of Amiel [11] who suggested deriving this temperature referred as $T^{*}$ from the wall superheat temperature $\left(T_{\mathrm{w}}-T_{\mathrm{b}}\right)$ and the Leidenfrost temperature $T_{1}$

$T^{*}=\frac{T_{\mathrm{w}}-T_{\mathrm{b}}}{T_{1}-T_{\mathrm{b}}}$

$T^{*}$ takes a negative value if the wall temperature is lower than the boiling point. It is higher than 1 when this temperature is above the Leidenfrost temperature. By definition, the heat flux exchanged between the wall and the droplet is minimal at the Leidenfrost temperature. The existence of such a minimum comes from the formation of a vapour cushion that prevents any direct contact between the droplet and the wall surface. Contrary to the boiling temperature, the Leidenfrost temperature does not depend solely on the nature of the liquid at a given external pressure. It is also a function of the wall roughness, since the roughness tends to increase the number of nucleation sites [22]. To simplify, the Leidenfrost temperature is fixed here at a constant value. This assumption is justified as the surface roughness, the ambient pressure, the liquid and wall materials were all kept constant during the experiments. For injected moving drops, their momentum increases the force towards the wall and the vapour cushion needs to be thicker to avoid the droplet to impinge the wall, leading to an increase of the Leidenfrost temperature [23]. However, these effects do not change significantly the Leidenfrost temperature in the range of droplet velocity encountered in the present study. For this reason and in the absence of available model, this effect is not considered in the following and it will be assumed that the Leidenfrost temperature has a constant value equal to $220^{\circ} \mathrm{C}$.

When the wall temperature is below the Leidenfrost temperature, the wall temperature in Eq. (7) is replaced by the effective contact temperature that accounts for the local cooling of the wall surface by the droplets. The use of the contact temperature has been suggested by Cossali et al. [7] to describe the thermally induced secondary atomization of impacting droplets. This temperature is defined from the thermal effusivities $\varepsilon=\sqrt{\lambda \rho C_{\mathrm{p}}}$ of the wall $\left(\varepsilon_{\mathrm{w}}=15,640 \mathrm{~J} / \mathrm{K} / \mathrm{m} / \mathrm{s}\right)$ and the liquid $\left(\varepsilon_{1}=1580 \mathrm{~J} / \mathrm{K} / \mathrm{m} / \mathrm{s}\right)$

$T_{\mathrm{w}}=\frac{\varepsilon_{\mathrm{w}} T_{\mathrm{th}}+\varepsilon_{\mathrm{l}} T_{1}}{\varepsilon_{\mathrm{w}}+\varepsilon_{\mathrm{l}}}$

In the latter expression, $T_{\text {th }}$ denotes the wall temperature before that a droplet impinges onto its surface. Presently, $T_{\text {th }}$ will be assumed to be the temperature measured by the thermocouple in the core of the solid block. Similarly, $T_{1}$ corresponds to the droplet temperature before the impact which is approximated here by the liquid injection temperature. Eq. (8) comes from the conservation of the heat flux at the interface between the droplet and the wall when neglecting any thermal contact resistance. The heat flux density entering into each medium is actually proportional to their effusivity [24].

The outcomes of the droplet impact are presented in Fig. 7 in a $T^{*}-K$ diagram. All types of rebounds are gathered here in a single category and three regions corresponding to the deposition, the bouncing and the splashing regimes can be clearly identified. As expected, the Leidenfrost temperature plays a central role in the droplet/wall interaction. $T^{*}=1$ appears actually as an upper limit above which the deposition regime cannot exist. It is well known that a transition exists between deposition and splashing for a cold surface. In the present study, $K$ does not exceed 1700 which explains probably why splashing is not observed for $T^{*}<1$. For a cold surface $\left(T^{*}<0\right)$, the value of $K$ corresponding to the transition between deposition and splashing is actually close to 3000 [12]. Additionally, it can be noticed that the fron- 


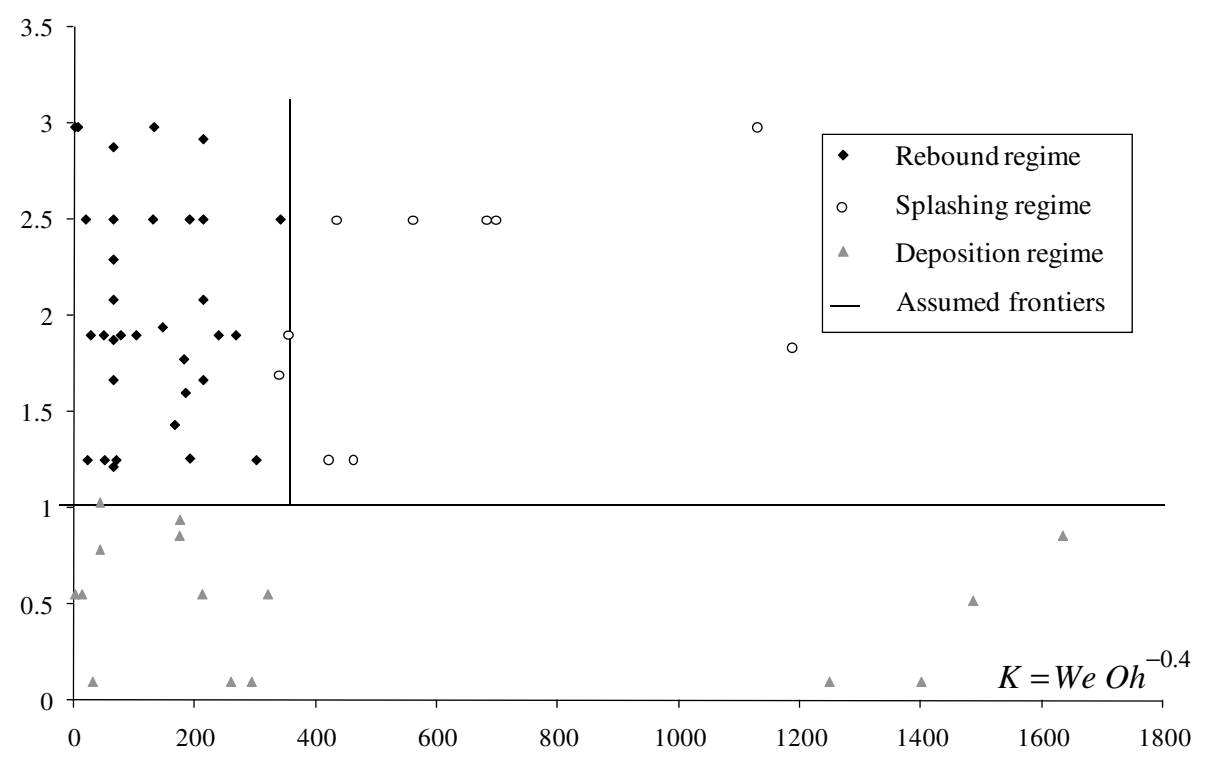

Fig. 7. Mapping of the different impact regimes in a $T^{*}-K$ diagram.

tier between the bouncing and the splashing regimes is a unique function of $K$ as long as $T^{*}$ is higher than 1 . A critical number $K_{C}$ equal to about 400 can be proposed to describe the reboundsplashing transition which is rather comparable to the value of 450 obtained by García Rosa et al. [12] in the case of ethanol and isooctane droplets injected under similar conditions.

\section{Study of the droplet heating}

The droplet temperature was measured with the help of the above-mentioned two-colour laser-induced fluorescence technique (see Section 3.2). The emphasis was placed on the characterization of droplet temperature very near the impact location. Attempts were made to quantify the variation of the liquid temperature resulting from the interaction between the droplets and the hot wall surface. This section presents measurements that were obtained in the case of three different impact regimes: the deposition regime, the bouncing and splashing regimes. A large number of experiments were carried out to test the influences of the wall temperature and the incident angle, while the droplet size and velocity as well as their injection frequency were maintained constant. The droplet diameter was about $106 \mu \mathrm{m}$ as estimated by the measurement of the liquid flow rate at the outlet of the injector. The value of the velocity derived from the observation of the images is about $9.1 \mathrm{~m} / \mathrm{s}$ and the injection frequency is fixed at $10 \mathrm{kHz}$. Additionally the camera is used to characterize the droplet angle of incidence and control their velocity. For all the measurements carried out on that droplet stream, pictures taken by the camera reveal that the droplet change in size is rather limited from the time they leave the generator until they impact the heated surface. However, the determination of the droplet size from the camera images is rather inaccurate. Given the magnification of the camera, a pixel corresponds to about $10 \mu \mathrm{m}$. Additionally the size estimation would also be sensitive to the level of the back lighting and the camera depth of field. Regarding the temperature droplet, no significant variation of the droplet temperature is noticed before they reach the plate. The time that the droplets spend in the air plume (generated by the natural convection from the plate) is indeed rather limited (in the order of $1 \mathrm{~ms}$ ) since the droplet stream is oriented perpendicular to the longer edge of the plate.

\subsection{Bubble boiling deposition regime}

The bubble boiling regime was first studied in order to test the reliability of the temperature measurement technique. The wall was maintained at a temperature of $160{ }^{\circ} \mathrm{C}$ and the incident angle was adjusted at $30^{\circ}$. The resulting liquid film which has a length of about $1 \mathrm{~cm}$ is large compared with the droplets. Attempts to operate with other wall temperatures demonstrated the difficulties to achieve perfectly stationary conditions. A perfect balance between the incoming liquid flow and the liquid vapourization is indeed required to insure the steadiness of the liquid film. For a wall temperature below $160^{\circ} \mathrm{C}$, the liquid film never stopped growing and eventually overflowed out of the plate. When the wall temperature is above $160^{\circ} \mathrm{C}$, a vapour layer formed intermittently at the interface with the liquid and induced unpredictable sliding of the liquid film and eventually its breaking. Fig. 8 depicts the distribution of the liquid temperature in a vertical section of the liquid film containing the axis of the droplet stream. The origin $x=0$ refers to the position of the droplet impingement. Temperature was measured at 0.2, 0.45, and $0.65 \mathrm{~mm}$ from the wall surface. It was not possible to measure the temperature closer to the wall since the beam diameter is about $150 \mu \mathrm{m}$. Due to the presence of vapour bubbles, the thickness of the liquid film was difficult to estimate. However, the fluorescence data give some insight about its possible value. The fluorescence signal is almost constant at 0.2 and $0.45 \mathrm{~mm}$; while it is a succession of individual events at $y=0.65 \mathrm{~m}$. This seems to indicate that the probe volume of the fluorescence is outside of the liquid film at $0.65 \mathrm{~mm}$ and that the thickness of the liquid film is bounded between 0.45 and $0.65 \mathrm{~mm}$. Out of the liquid film, the fluorescence signal is provided from liquid samples ejected by the bursts of the vapour bubbles. As expected, whatever the position in the liquid film the measured temperature is rather close to the boiling temperature of water (Fig. 8). However, a thermal gradient can be noticed, the liquid being colder close to the wall surface. Although boiling appears at the wall surface, the measured temperature is lower than the boiling point at that place. In fact, boiling occurs rather intermittently and remains localized, while the measured temperature corresponds to an average over the entire volume measurement and over a period of acquisitions of a few seconds. Measurements 

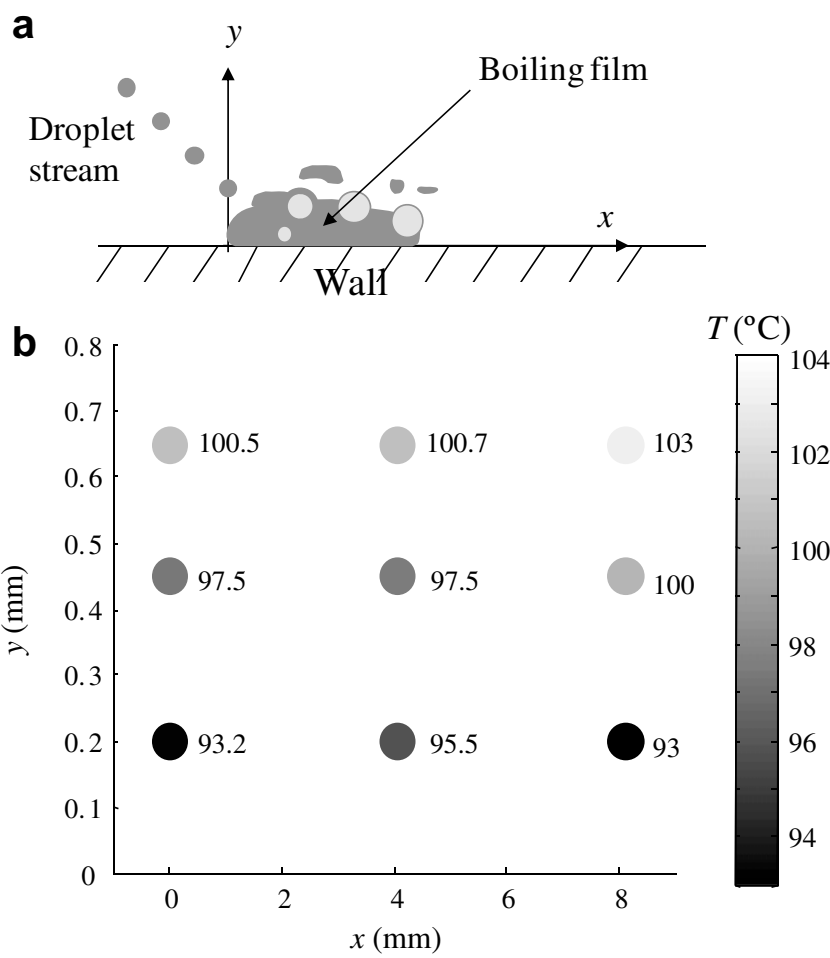

Fig. 8. Temperature distribution in a boiling liquid film $\left(T_{\mathrm{w}}=160^{\circ} \mathrm{C}, D=106.6 \mu \mathrm{m}\right.$, $V=9.1 \mathrm{~m} / \mathrm{s}, f=10 \mathrm{kHz})$ ((a) geometrical arrangement of the measurement, (b) scattering of the data). $x$ and $y$ are the horizontal and vertical axes $(x=0, y=0)$ denotes the impact location of the droplets and the $x$-axis is oriented along the direction of the droplet stream.

seem to indicate that the cold liquid of the impinging droplets is mixing with the hot liquid film preferentially in the regions that are the closest to the wall.

\subsection{Film boiling, splashing and rebound regimes}

\subsubsection{Influence of the angle of incidence}

The influence of the incident angle was more particularly investigated. In this section, the emphasis was placed on the droplet heating during an impact. To that end, the droplet temperature was measured at two locations: before the droplet impact and just after it. In the two cases, the measurement volume was positioned at $0.5 \mathrm{~mm}$ from the wall (Fig. 9). When a splashing occurs, only a limited part of the secondary droplets passes through the measurement volume. To make this sample of droplets as representative as possible, the measurement volume was placed in the main direction of the droplets emission if one can be determined. The results presented in this section were obtained at a wall temperature of $400{ }^{\circ} \mathrm{C}$ which is far above the Leidenfrost temperature of water. The droplets were injected at room temperature (about $22^{\circ} \mathrm{C}$ ), but a slight increase of the injection temperature (in the order of $4{ }^{\circ} \mathrm{C}$ ) could be found because of the proximity between the injector and the heated wall. No significant variation of the droplet temperature was noticed in the distance between the droplet generator and the plate. The time that the droplets spend in the air plume (generated by natural convection from the plate) is rather limited in the order of $1 \mathrm{~ms}$ since the droplet stream is oriented parallel to the smaller edge of the plate which is $2 \mathrm{~cm}$ long. Measurements were performed for incident angles ranging from $10^{\circ}$ to $80^{\circ}$. The case of a normal impact was not considered because of the need to discriminate between primary and secondary droplets that may also coalesce in this configuration. For incident angles lower than $47^{\circ}$, the result of an impact is a rebound, whereas the regime observed beyond is a splashing. Fig. 10 shows the droplet change in temperature during an impact as a function of the angle of incidence $\alpha$. In this figure, it can be noticed that the heating of the droplets is all the more important than the angle of the droplet stream is high. This heating reaches about $40^{\circ} \mathrm{C}$ in the case of an angle of $80^{\circ}$. The droplet variation in temperature increases continuously, i.e. no discontinuity between the bouncing and the splashing regimes can be pointed out. For the lowest values of the angle of incidence, the droplet change in temperature rises rapidly and gradually reaches a plateau above an angle of incidence of $50^{\circ}$, which corresponds also to the appearance of the splashing regime. Additional measurements are required to determine whether the appearance of this plateau is only related to the incident angle or if it is due to the nature of the impact regime. It would have been also interesting to measure the evolution of the droplet diameter during the impact especially in the case of the rebound regime. However, the measurement of the droplet size is not accurate enough with the current imaging system. It is only possible to state that evaporation does not exceed $10 \mu \mathrm{m}$ which is the limit of the spatial resolution of the camera. The present measurements illustrate the importance of the normal component of the velocity in the heating of the droplets. Considering that the droplet velocity was kept constant during these experiments, an increase in the incident angle is actually equivalent to an increase in the normal velocity and hence in the Weber number defined in Eq. (5). The observed trends can be qualitatively explained. It is well known

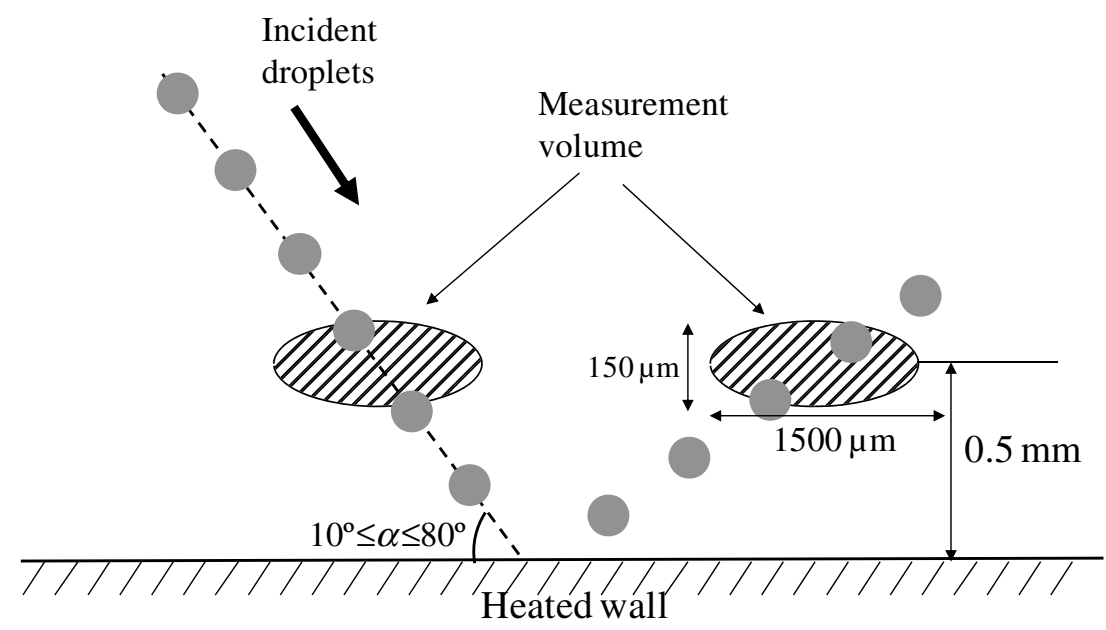

Fig. 9. Location of the measurement volume in the characterization of the droplet variation in temperature during an impact. 


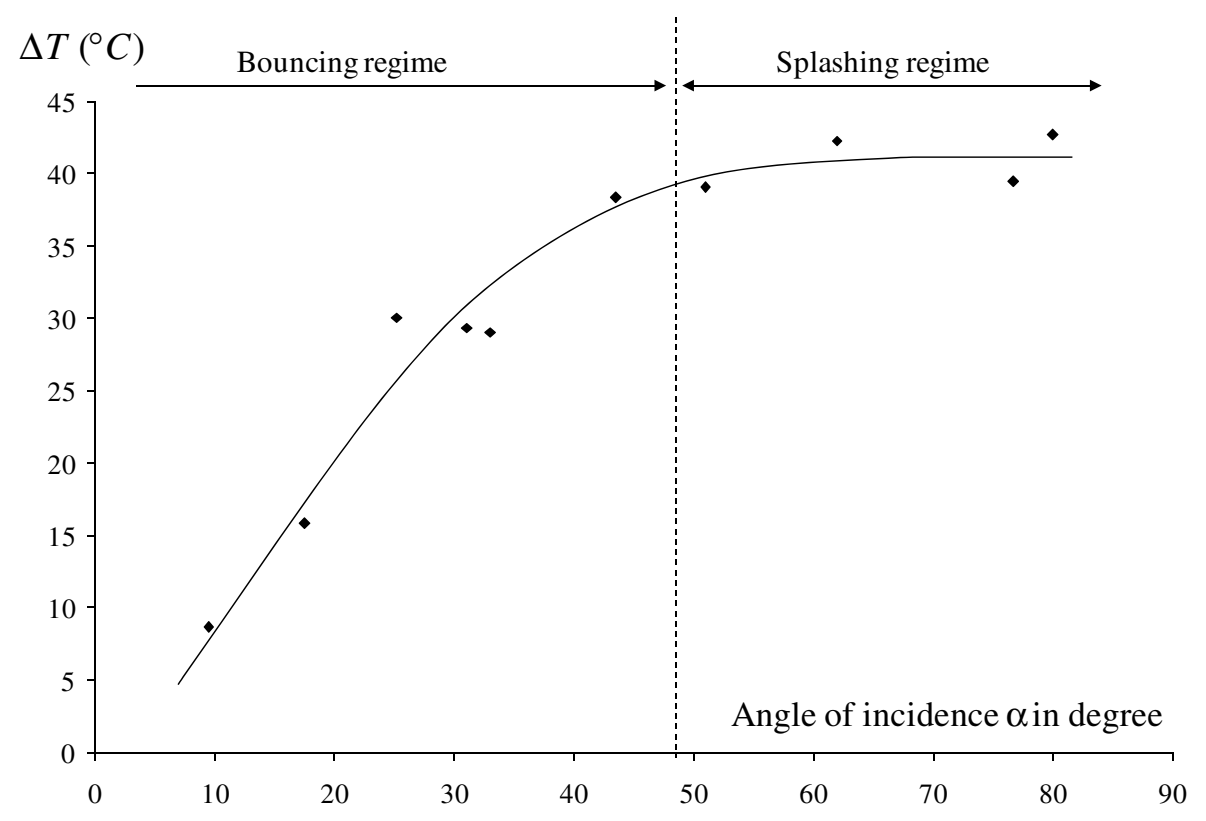

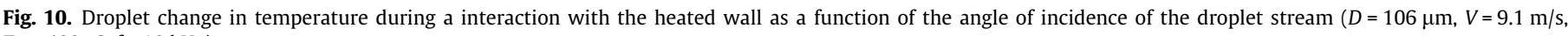
$T_{\mathrm{w}}=400^{\circ} \mathrm{C}, f=10 \mathrm{kHz}$ ).

that the maximum spreading radius of the droplet increases with the Weber number [25] and consequently the surface area of the thermal transfer from the wall to the droplet. An increase in the normal velocity can also reduce the thickness of the vapour layer separating the liquid and the wall. This layer acts as a thermal insulator which decreases the thermal conduction from the wall to the droplet even more that it is thick. Above a certain angle of incidence, the kinetic energy of the droplet may be large enough for the droplet to cross this vapour layer and come into direct contact with the wall. The residence time of the droplet in the vicinity of the wall should also have an important influence on the heat transfer to the droplets. However, the residence time depends mainly on the droplet diameter and very weakly on the normal velocity [26]. For this reason, this parameter cannot be cited here to explain the differences of heating observed among the tested conditions since the droplet size was maintained constant during the experiments.

\subsubsection{Influence of the wall temperature}

A set of similar experiments was performed in order to highlight the influence of the wall temperature. The wall temperature has been changed from 250 to $440{ }^{\circ} \mathrm{C}$ and two angles of incidence

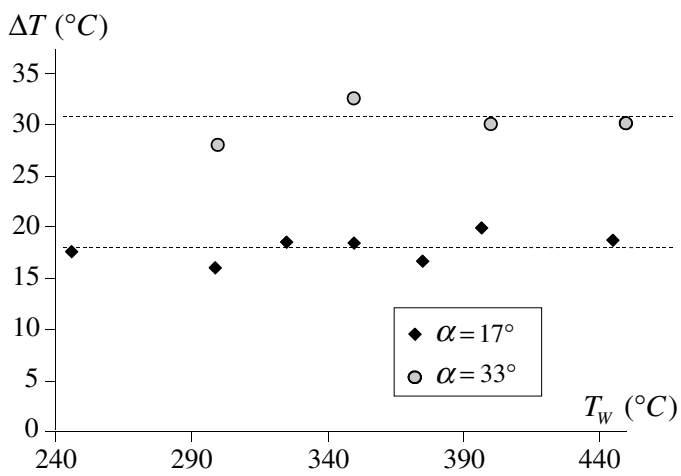

Fig. 11. Temperature of the droplets after their rebound onto the heated wall as a function of the wall temperature in the case of two angles of incidence $17^{\circ}$ and $33^{\circ}$ $\left(D=106 \mu \mathrm{m}, V=9.1 \mathrm{~m} / \mathrm{s}, T_{\text {inj }}=28^{\circ} \mathrm{C}, f=10 \mathrm{kHz}\right)$. corresponding to $\alpha=17^{\circ}$ and $\alpha=33^{\circ}$ were tested. Results are therefore limited to the film boiling domain and more especially to the rebound regime. Fig. 11 shows the droplet increase in temperature during a rebound onto the wall as a function of the wall temperature. In this experiment, the droplets are injected at about $28^{\circ} \mathrm{C}$. As expected, the droplet heating is higher with an incident angle of $33^{\circ}$ than with one of $17^{\circ}$. It can be noticed that the change in temperature seems fairly independent on the wall temperature in the investigated range of experimental conditions. This observation is in a good agreement with the current state of knowledge. For instance, no significant effect of wall temperature on the maximum spreading radius of rebounding droplets has been reported for a dry wall with a temperature above the Leidenfrost point. When increasing the wall temperature, the thickening of the vapour layer separating the wall and the droplet is too limited to have a significant effect on the heat transferred to the droplet. It is widely accepted that the heat flux increases very slowly when the wall temperature exceeds the Leidenfrost temperature like in the case of pool boiling.

\section{Conclusion}

The two-colour laser-induced fluorescence thermometry has been used to characterize accurately the heating of droplets interacting with a hot wall. Measurements indicate that the droplet increase in temperature during an impact can reach almost $40{ }^{\circ} \mathrm{C}$ in the case of a splashing whereas it is more limited in the case of a rebound. The droplet heating is all the more important than the normal component of the velocity is high, but no significant effect of the wall temperature on the droplet heating can be pointed out provided that the impact regime is a rebound. The droplet heating during an impact which is large enough to be captured by the experimental technique certainly deserves to be taken into account in a description of the interactions between the droplets and a high-temperature wall. In the near future, further investigations using the two-colour LIF thermometry should help the modelling of the droplet heating as a function of the impact regime. The use of the two-colour LIF thermometry to address the issue of spray cooling seems to have interesting prospects. In particular, 
valuable information about the energy balance of the interaction process could be obtained, if the technique is combined to other experimental methods such as the measurement of the droplet sizes to follow its evaporation or the characterization of the heat flux removed from the wall. The use of the droplet exit temperature (instead of the injection temperature) would be particularly well indicated to evaluate the dimensionless numbers on which the description of the droplet dynamic is based. Nonetheless, further studies will be necessary to characterize the droplet change in temperature during an impact and to propose a proper reference temperature. The most important effect is probably on the liquid viscosity which decreases quite strongly with temperature. At $40{ }^{\circ} \mathrm{C}$, water viscosity $\left(0.65 \times 10^{-3} \mathrm{~Pa} \mathrm{~S}\right)$ is decreased by $35 \%$ compared to $20^{\circ} \mathrm{C}$, which results in a decrease of the Ohnesorge number and an increase of about $15 \%$ of the critical value of the Mundo number $K$ (460 instead of about 400 ).

\section{References}

[1] J.D. Bernardin, I. Mudawar, Film boiling heat transfer of droplet streams and sprays, Int. J. Heat Mass Transfer. 40 (2) (1997) 2579-2593.

[2] B.W. Webb, C.F. Ma, Single-phase liquid jet impingement, Adv. Heat Transf. 26 (1995) 105-217.

[3] S.C. Yao, K.Y. Cai, The dynamics and Leidenfrost temperature of drops impacting on a hot surface a small angles, Exp. Therm. Fluid Sci. 1 (1988) 363-371.

[4] R. Rioboo, C. Tropea, M. Marengo, Outcomes from a drop impact on solid surfaces, Atomization Sprays 11 (2) (2001) 155-165.

[5] G.I. Taylor, Oblique impact of a jet on a plane surface, Philos. Transfer. R. Soc Lond. A 260 (1966) 96-100.

[6] S. Sikalo, M. Marengo, C. Tropea, E.N. Ganic, Analysis of impact of droplets on horizontal surfaces, Exp. Therm. Fluid Sci. 25 (2002) 503-510.

[7] G.E. Cossali, M. Marengo, M. Santini, S. Fest, Effect of wall effusivity on thermally induced secondary atomisation of single drop impacting onto a tilted surface, in: Proceedings of the 10th International Conference on Liquid Atomization and Spray System, Kyoto, Japan, 2006.

[8] K. Anders, N. Roth, A. Frohn, The velocity change of ethanol droplets during collision with a wall analysed by image processing, Exp. Fluids 15(1993) 91-96.

[9] J.D. Bernardin, Leidenfrost Point and Film Boiling Heat Transfer of Single Droplets and Sprays, Ph.D. Thesis, Purdue University, 1996.
[10] L.M.J. Wachters, N.A.J. Westerling, The heat transfer from a hot wall to impinging water drops in the spheroidal state, Chem. Eng. Sci. 21 (1966) 10471056.

[11] C. Amiel, Application de techniques optiques à l'étude du comportement dynamique et thermique de gouttes en interaction avec une paroi chauffée, Ph.D. Thesis, École Nationale Supérieure de l'Aéronautique et de l'Espace, Toulouse, 2002.

[12] N. García Rosa, P. Villedieu, J. Dewitte, G. Lavergne, A new droplet-wall interaction model, in: Proceedings of the 10th International Conference on Liquid Atomization and Spray System, Tokyo, Japan, 2006.

[13] K.J. Baumeister, F.F. Simon, Leidenfrost temperature - its correlation for liquid metals, cryogens, hydrocarbons and water, Trans. ASME J. Heat Transfer. 95 (1973) 166-173.

[14] H. Ulmke, M. Mitschke, K. Bauckhage, Piezoelectric single nozzle droplet generator for production of monodisperse droplets of variable diameter, Chem. Eng. Technol. 24 (1) (2001) 69-70.

[15] A. Frohn, N. Roth, Dynamics of Droplet, Springer, Berlin, 2000.

[16] P. Lavieille, F. Lemoine, G. Lavergne, M. Lebouché, Evaporating and combusting droplet temperature measurements using two-color laserinduced fluorescence, Exp. Fluids 31 (1) (2001) 45-55.

[17] G. Castanet, P. Lavieille, M. Lebouché, F. Lemoine, Measurement of the temperature distribution within monodisperse combusting droplets in linear stream using two colors laser-induced fluorescence, Exp. Fluids 35 (2003) 563-571.

[18] J. Van Beeck, M. Riethmuller, Non-intrusive measurements of temperature and size of single falling raindrops, Appl. Opt. 34 (1995) 1633-1639.

[19] K.J. Baumeister, F.F. Simon, R.E. Henry, Role of the surface in the measurement of the Leidenfrost temperature, Augmentation Convective Heat Mass Transfer. ASME (1970) 91-101.

[20] C.H.R. Mundo, M. Sommerfield, C. Tropea, Droplet-wall collisions: experimental studies of the deformation and break-up process, Int. J. Multiphase Flow 21 (2) (1995) 151-173.

[21] G.E. Cossali, A. Coghe, M. Marengo, The impact of a single drop on a wetted solid surface, Exp. Fluids 22 (1997) 463-472.

[22] J.D. Bernardin, I. Mudawar, An experimental investigation into the relationship between temperature-time history and surface roughness in the spray quenching of aluminium parts, ASME J. Mater. Technol. 118 (1) (1996) 127134.

[23] M. Rein, Interactions between drops and hot surfaces, Drop-Surface Interactions, Springer, Wien, New York, 2002, ISBN 3-211-83692-6.

[24] J.F. Sacadura, Initiation aux transferts thermique, Technique et Documentation, 1993.

[25] A. Karl, A. Frohn, Experimental investigation of interaction processes between droplets and hot walls, Phys. Fluids 12 (4) (2000) 785-796.

[26] R.-H. Chen, S.-L. Chiu, T.-H. Lin, Resident time of a compound drop impinging on a hot surface, Appl. Therm. Eng. 27 (2007) 2079-2085. 